\title{
Analgesia for Moderate Chronic Non-Cancer Pain : Low Dose Transdermal Buprenorphine a Novel Option in Mexico
}

\author{
Joseph V. Pergolizzi ${ }^{1,2,3^{*}}$, Robert B. Raffa ${ }^{4}$, Miguel Angel Ruiz Ibán ${ }^{5}$, Gianpietro Zampogna ${ }^{6}$, Robert \\ Taylor $^{6}$
}

${ }^{1}$ Department of Medicine, Johns Hopkins University School of Medicine, Baltimore, MD

${ }^{2}$ Department of Pharmacology, Temple University School of Medicine, Philadelphia, PA

${ }^{3}$ Association of Chronic Pain Patients, Houston, TX

${ }^{4}$ Department of Pharmaceutical Sciences, Temple University School of Pharmacy, Philadelphia, PA

${ }_{5}^{5}$ Shoulder and Elbow Unit. Hospital Universitario Ramóny Cajal, Madrid, Spain

${ }^{6}$ NEMA Research, Bonita Springs, FL

"Corresponding author: Joseph V. Pergolizzi, MD, Department of Medicine, Johns Hopkins University School of Medicine, Baltimore, E-mail:drpergo@gmail.com

\begin{abstract}
Chronic non-cancer pain is prevalent in Mexico and its pharmacologic treatment requires clinicians to balance the risks and benefits of various analgesic agents. NSAIDs and paracetamol (acetaminophen) can be effective for mild to moderate pain, but safety considerations place limitations on their use. Opioids are safe and effective, but have opioid-associated side effects plus the potential for abuse. Against this background, it is important to appraise other options with regard to favorable efficacy and safety - such aslow-dose transdermal buprenorphine. Buprenorphine, both in transdermal and oral formulations, has been available in Mexico for a number of years yet just recently a Low-Dose Transdermal Patch formulation has been available for the management chronic non-cancer pain of moderate intensity in adults. Buprenorphine is an opioid agent with a unique pharmacological profile, such that it has a ceiling effect for respiratory depression, but no ceiling effect for analgesia. It can be used without dose adjustment in the elderly and in patients with impaired kidney function (unique among opioids). Its small lipophilic molecule makes it well suited to transdermal formulations, which offer steadystate round-the-clock analgesia after three days with clinical convenience and easier patient compliance. Buprenorphine is an effective analgesic in chronic non-cancer pain patients, and its good tolerability and lower abuse potential may make lowdose transdermal buprenorphine appropriate for a broad range of patients.
\end{abstract}

\section{Intraduction}

Pain medicine has come a long way since the now-famous three-rung "pain ladder" proposed by the World Health Organization (WHO) in 1988. The WHO pain ladder recommended treating cancer pain based solely on intensity and although it allowed for combination therapy and adjuvant agents, it stopped short of specifying them or offering guidance on their use ${ }^{[1]}$. It also did not address non-cancer pain. Since 1988, novel analgesic products and new formulations of existing products have come to market and our understanding of the multimechanistic nature of many pain syndromes has increased. For example, the American Geriatric Association recently changed its guidance for pain control in the elderly by advocating opioids be used as first-line agents with NSAIDs reserved for short-term use to manage pain exacerbations and flares ${ }^{[2]}$.

Yet, treating chronic nonmalignant pain remains challenging in that multimodal therapy is often required, and the pharmacological regimen should be individualized to meet the patient's needs. In Mexico, there are two board groups of
Received date: May 4, 2015

Accepted date: February 22, 2016

Published date: February 24, 2016

Citation: Pergolizzi, J., et al. Analgesia for Moderate Chronic Non-Cancer Pain: Low Dose Transdermal Buprenorphine a Novel Option in Mexico. (2016) J Anesth Surg 3(1): 96- 101.

DOI: $10.15436 / 2377-1364.16 .022$

Keywords: Transdermal low dose buprenorphine; Chronic pain; Opioids; Buprenorphine; Chronic non-cancer pain

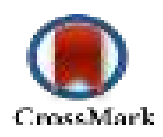

pain medicine prescribers: those who are experienced with buprenorphine, including a high dose transdermal system patch (TRANSTEC ${ }^{\text {TM}) ~ f o r ~ w h o m ~ t h e ~ f a v o r a b l e ~}$ clinical attributes of buprenorphine are well known; and those who have not or do not currently prescribe buprenorphine. In this setting, low-dose transdermal buprenorphine is worthy of consideration.

\section{The role of opioid analgesics}

Opioid agents are effective pain relievers, but haveassociated side effects such as constipation, dizziness, somnolence, nausea, etc., plus carry an abuse 
potential $^{[3]}$. Familial, social, legal, public health, and regulatory concerns make some prescribers reluctant to consider opioids, contributing to "opiophobia"[4] . Other impediments to longterm opioid therapy include possible development of tolerance (where the patient requires increasing doses of the same agent to maintain the equivalent level of analgesia) $)^{[5]}$ and opioid-induced hyperalgesia $(\mathrm{OIH})$, a paradoxical condition in which opioid use appears to lower the pain threshold ${ }^{[6]}$. These drawbacks have prompted many clinicians in Mexico to avoid using opioids for pain management. It has been estimated that only about one in a thousand registered doctors use major opioids in Mexico ${ }^{[7]}$.

Yet, in the last years, significant concerns have arisen for the use of other therapeutic agents for long term pain management in patients with chronic non-cancer pain; in particular recent high quality dataon the cardiovascular safety of both paracetamol ${ }^{[8]}$ and NSAIDs ${ }^{[9]}$ has put into question the sustained use of these drugs in patients with relevant cardiovascular risk. This has caused many clinicians, including those formerly reluctant about the use of opioids, to reconsider their use, particularly in the low-dose transdermal formulation.

\section{The case for buprenorphine}

Buprenorphine exerts effective analgesic action for multiple pain types, including bone pain, thermal pain (heat and cold-pressor pain), and neuropathic pain and,although it does not have a direct anti-inflammatory effect, it is active against inflammatory and visceral pain ${ }^{[10]}$. The buprenorphine molecule has very high affinity (in the sub-nanomolar concentration range) for the $\mu$-opioid receptor and the other seven-transmembrane $\mathrm{G}$ protein-coupled opioid receptors (delta and kappa), but its intrinsic activity at $\delta$ and $\kappa$ sites is minimal, so its analgesic activity is thought to be mediated primarily by $\gamma$-opioid receptors. Buprenorphine exerts a lasting antihyperalgesic effect ${ }^{[11]}$. In terms of clinical analgesic efficacy, buprenorphine is comparable to morphine, the "gold standard" of opioids ${ }^{[12]}$

Among the opioid agents, buprenorphine may be described as having a unique pharmacology ${ }^{[10]}$. Although often described as a partial agonist based on in vitro data,this term is misleading, because buprenorphine exerts a full analgesic effect in most preclinical studies ${ }^{[13,14]}$ andin humans, radio-labeling studies demonstrate that full analgesia can be produced with less than $100 \%$ occupancy of the $\mu$-opioid receptor, which is the definition of a full agonist ${ }^{[15]}$. This is likely due to the contribution of other analgesic mechanisms ${ }^{[8,16]}$.

The dose-response curve for buprenorphine over the therapeutic range of the drug is roughly linear (doses 0.2 to $\leq$ $7 \mathrm{mg}$ ) and at higher doses ( 8 to $32 \mathrm{mg}$ ) continues to offer increased analgesia ${ }^{[17]}$. On the other hand, buprenorphine demonstrates a ceiling effect for respiratory depression (a ceiling effect is the point at which no further effect is achieved, despite increasing doses), unique among the other opioid analgesics ${ }^{[10]}$. Since respiratory depression is a particularly serious and potentially life-threatening adverse event, this characteristic makes buprenorphine an important agent in the opioid armamentarium.

Buprenorphine is also unique among the opioids in that it alone can be safely used in patients with compromised renal function.Buprenorphine, when given intravenously, is mainly secretedinto bile and excreted in urine. Unlike other opioids, the half-lives of the drug and metabolites do not increase in persons with compromised renal function, such as (but not limited to) the elderly ${ }^{[18]}$. In fact, the analgesic effects and side effect profiles of buprenorphine do not vary in older ( $>65$ years) versus younger $(<50$ years $)$ patients ${ }^{[19]}$. In an open-label observational study of elderly chronic non-cancer pain patients (mean patient age 73 years, $n=891$ ), transdermal buprenorphine reduced pain intensity by a score of $5.1(1.7 \pm 1.3$ from $6.8 \pm 1.5)$, a $76 \%$ improvement on the rating scale ${ }^{[20]}$.

Like other opioid agents, buprenorphine may expose patients to potentially serious pharmacokinetic drug-drug interactions when used with other agents. In particular, such drugdrug interactions may occur when buprenorphine is taken concomitantly with cytochrome (CYP)450-3A4 inhibitors (azoles, macrolides, non-nucleoside reverse transcriptase inhibitors, and protease inhibitors, among others) as well as CYP450-3A4 inducers (phenobarbital, carbamazepine, phenytoin, rifampicin) ${ }^{[21]}$. Buprenorphine may have a potentially life-threatening effect when administered to patients with severely compromised hepatic function when combined with high-dose benzodiazepine therapy ${ }^{[21]}$. In general, buprenorphine should be used with extreme clinical caution (if at all) in patients taking benzodiazepines, central nervous system depressants, certain antidepressants, anxiolytics, and other opioids. Furthermore, alcohol should be avoided with buprenorphine use ${ }^{[21]}$.

\section{Clinical efficacy of low dose transdermal buprenorphine}

Buprenorphine is available as a transdermal system for pain relief; its long duration of action and lipophilic molecule make it well suited to this route of administration. In Mexico, Low Dose Transdermal Buprenorphine (LDTB) is available in $5 \mu \mathrm{g} / \mathrm{h}$ and $10 \mu \mathrm{g} / \mathrm{h}$ systems. The patch allows for continuous delivery of buprenorphine for seven days, achieving steady state by the third day following the initial patch application. An equipotency ratio of oral morphine to transdermal buprenorphine has been proposed as 1:75, but other recent sources suggest 1:70 or $1: 115^{[22-24]}$.

A review of transdermal buprenorphine in the treatment of chronic non-cancer pain found it was significantly more effective than placebo in reducing pain and was well tolerated by patients ${ }^{[25]}$. A double-blind parallel-group study of 588 chronic non-cancer pain patients found transdermal buprenorphine to be significantly more effective in reducing pain than placebo, with significantly reduced rescue medication consumption versus placebo. It was well tolerated by patients, with the most common adverse events being pruritus at application site, headache, and somnolence $(9.3 \%, 3.9 \%, 2.3 \% \text {, respectively })^{[26]}$.

The transdermal formulation may offer certain clinical advantages in that it reduces compliance issues in outpatients and offers convenience to both patients and clinicians. In a postmarketing study from Poland $(\mathrm{n}=4030), 70.1 \%$ of chronic cancer and non-cancer pain patients treated with transdermal buprenorphine intended to continue treatment with the transdermal system $^{[27]}$ (Figure 1). In a larger postmarketing study in Europe $(n=13,179), 80 \%$ reported good or very good pain relief at the conclusion of the study and only $5 \%$ of patients in this study discontinued transdermal buprenorphine for lack of efficacy ${ }^{[28]}$. 


\section{Pain relief}

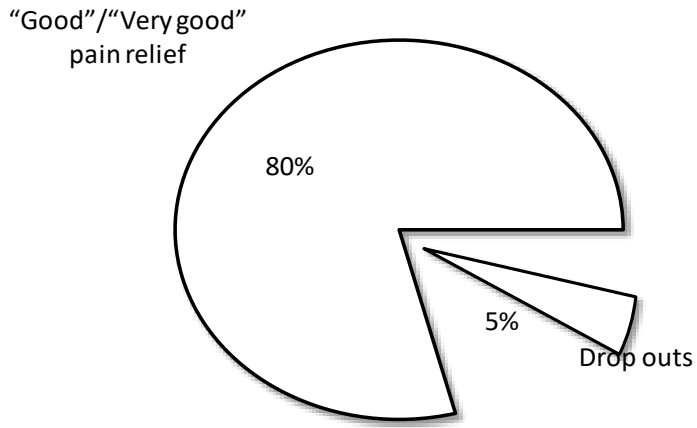

Figure 1: Analgesic efficacy of transdermal buprenorphine, indicated by "good" or "very good" pain relief in a post-marketing study of 13,179 patients.

The muskuloskelletal system is the most common source of chronic non-malignant pain and the efficacy of LDTP has been extensively evaluated in this group of patients. In a non-inferiority study comparing transdermal buprenorphine to tramadol, shown in (Figure 2). 280 patients with moderate to severe musculoskeletal pain were randomized for eight weeks of treatment. Both treatment arms achieved significant pain reduction over baseline and the least squares mean difference of the change from baseline between the arms was 0.45 (95\% confidence interval, range -0.02 to 0.91 ) which met the predefined \pm 1.5 threshold for non-inferiority ${ }^{[29]}$.

\section{Patients' preference}

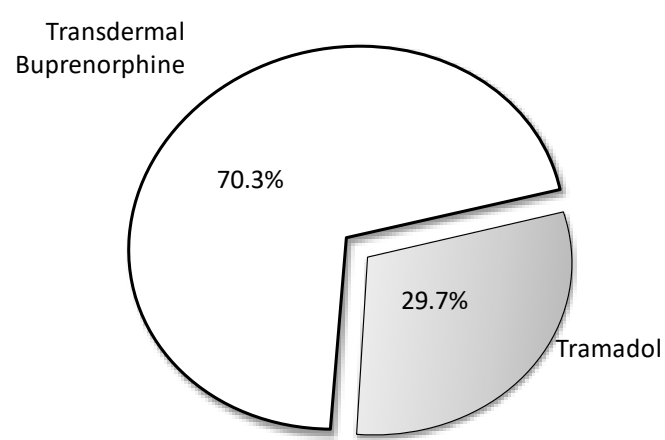

Figure 2: Patient preference of transdermal buprenorphine vs tramado in a study of chronic osteoarthritis pain

\section{Efficacy in osteoarthritis}

Osteoarthritis (OA) is considered one of the main causes of chronic non-malignant pain affecting 34\% of patients with chronic pain ${ }^{[30]}$. Buprenorphine is considered an excellent alternative for pain management in patients with osteoarthritis; in fact, osteoarthritis was the most frequently recorded indication (48.7\%) for LDTB was osteoarthritis in a large retrospective cohort study in England ${ }^{[31]}$. In a placebo controlled study of 311 patients suffering from knee or hip OA in which NSAIDs had not been effective, LDTP showed efficacy and good tolerability $^{[32]}$. In another study of 134 patients suffering from chronic OA pain, patients were randomized to receive a 12 -week treatment of LDTB or tramadol. Both agents were effective in reducing pain versus baseline and the use of rescue medication was similar in both groups. However, despite similar findings in terms of pain relief, quality of sleep and awakenings at night, $70.3 \%$ of patients in the study preferred transdermal buprenorphine $(95 \%$ confidence interval, 62 to 78$)^{[33]}$. In a study of hip or knee OA patients, 220 patients were randomized to be treated for 12 weeks with either a fixed-dose combination product of codeine plus acetaminophen or transdermal buprenorphine plus acetaminophen. Both treatments significantly reduced pain versus baseline, but the transdermal buprenorphine patients used significantly less rescue medication (ibuprofen, $\mathrm{p}=0.002)^{[34]}$.

\section{Efficacy in low back pain}

The impact that low back pain (LBP) has in the general population cannot be overstimated as it is a significant cause of disability and increased medical costs in the general population $^{[35]}$. LDTB has been proposed as an excellent alternative to deal with pain and loss of function in patients with LBP.Gordon et al ${ }^{[36]}$. Evaluated the efficacy of LDTB in patients 78 with LBP that had previously taken mayor opioids; in this placebo controlled randomized crossover clinical trial LDTP showed significant efficacy when compared to placebo with only $10 \%$ of patients withdrawing due to lack of efficacy. Interestingly, different authors have found in other placebo controlled randomized clinical trials that, in patients with LBP, LDTP has a positive impact in activities of daily living ${ }^{[37]}$, quality of life ${ }^{[38]}$ and sleep ${ }^{[39]}$ outcomes (Figure 3).

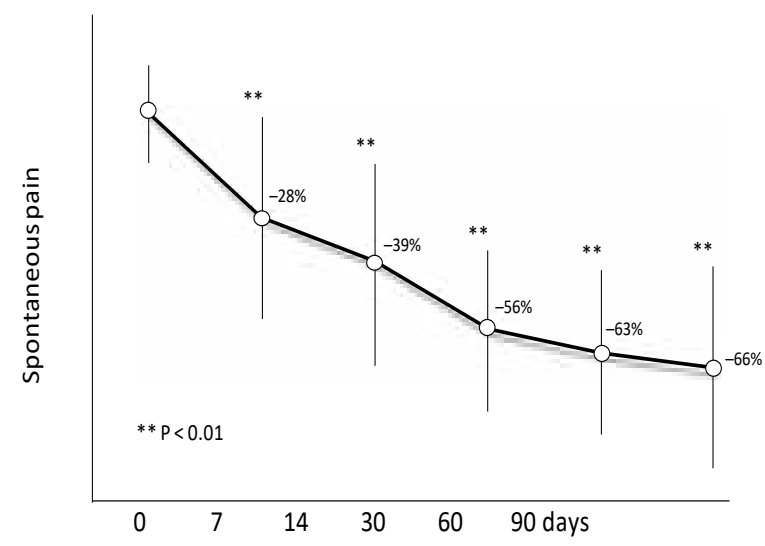

Figure 3: Reduction in pain intensity in patients over the age of 70 . Transdermal buprenorphine was administered for 3 months.

In a much larger randomized clinical trial Steiner et $\mathrm{al}^{[40]}$. recruited 660 patients with moderate to severe LBP and compared the efficacy and safety of LDTB versus oral oxycodone. They found that LDTB was both efficacious and safe in this population with a similar withdrawal rate due to side effects but lower constipation and headache rates.

\section{Abuse potential}

Clinicians sometimes are faced with treating pain in patients at risk for substance abuse or, in more extreme situations, patients with active addictions. Addictions to non-opioid substances may indicate a risk for opioid abuse $\mathrm{e}^{[41]}$. The literature reports on potential risk factors for opioid misuse and abuse, and screening tools ${ }^{[42-46]}$. Buprenorphine is considered to have lower potential for abuse than equianalgesic strong opioids ${ }^{[10]}$. In a double-blind, placebo-controlled, inpatient study of morphine-maintained substance abusers, an injection of buprenorphine resulted in little "good effect" reported by the subjects, 
who evidenced only a moderate interest in the drug ${ }^{[47]}$. This low "likeability" contributes to buprenorphine's lower abuse potential. Buprenorphine in other formulations (typically injections or sublingual products) is used as a first-line agent in opioid detoxification and maintenance programs, in part because of its relatively lower potential for abuse compared to other opioids ${ }^{[48]}$. In the US, the Drug Enforcement Agency (DEA) has classified buprenorphine as a Schedule III drug because it has less abuse potential than Schedule I (heroin) and Schedule II agents (morphine, oxycodone, fentanyl $)^{[49]}$. In Mexico, the regulatory body responsible for drug enforcement and control is the Comisión Federal Para la Protección contra Riesgos Sanitarios. It has classified buprenorphine as a psicotropic substance inside Fracción II, defined as a drug that has "certain therapeutic value, but can constitute a public health problem"; this situation makes buprenorphine a less-controlled drug than Fracción I drugs such as heroin or morphine ${ }^{[47]}$. When prescribing opioid agents, clinicians must be mindful not only of the risk presented to the patient but also to the community at large. Transdermal products are not,technically, "abuse-deterrent formulations," but the lowdose buprenorphine patch system makes it difficult to extract the drug for potential abuse.

\section{Tolerability of transdermal buprenorphine}

When the use of any drug is considered in a certain patient, the physician has to take into account both the efficacy and safety profile of the drug. When using opiods, safety concers are paramount as adverse effects are specially prevalent and are the most common cause of treatment withdrawal. A classical metaanalisys by Moore and McQuay showed that 21\% to 23\% of patients with chronic non-malignant pain that were started on opioids withdrew from their treatments due to side effects while lack of efficacy was the cause of withdrawal in only $5.6 \%$ to $7.4 \%$ of patients ${ }^{[0]}$. The most frequently reported adverse events associated with transdermal buprenorphine in the United States ( $\geq 5 \%$ ) were nausea, headache, pruritus at application site, dizziness, vomiting, constipation, somnolence, erythema at application site, dry mouth, and rash at the application site ${ }^{[25]}$.

When the safety profile of transdermal buprenorphine is evaluated, it seems to come out favourably when compared to other posology and therapeutic alternatives. A study that compared LDTB with sublingual buprenorphine in 246 patients with knee or hip OA showed that the general rate of adverse effects was lower with the transdermal formulation ${ }^{[33]}$. There were significant reductions in the most frequent side effects such as nausea ( $37 \%$ vs. $47 \%, p=0.035)$, dizziness $(27 \%$ vs. $41 \%, p=0.026)$ and vomiting $(18 \% \text { vs. } 29 \%, \mathrm{p}=0.039)^{[51]}$. Another study compared the efficacy and safety of LDTB and oral tramadol in 134 patients with OA and found similar decreases in pain levels but an increased satisfaction with treatment in the LDTB group, with significantly fewer withdrawals than in the tramadol group $(14.5 \%$ withdrawals with LDTB vs. $29.2 \%$ withdrawals with oral tramadol, $\mathrm{p}=0.032)^{[33]}$. Application site related side effects are a concern with transdermal systems as they might cause treatment withdrawal. A recent meta analysis has looked specifically at cutaneous problems with transdermal buprenorphine pooled the results of 16 studies with 6566 patients. It showed that the incidence of cutaneous problems was significant as these appeared in $23 \%$ of patients. Most of the cutaneous adverse events were not severe (98\%) and resolved upon patch site change or with local or systemic treatment. Only in $4.4 \%$ of cases, the application site related adverse effects caused product discontinuation $^{[52]}$. In a large postmarketing study of transdermal buprenorphine $(n=13,179), 22 \%$ of patients reported some form of adverse event ${ }^{[28]}$. These included nausea, dizziness, vomiting and constipation. In addition, of the 13,179 patients, only one case of respiratory depression was reported (which was not classified as serious).

\section{Use in the elderly population}

Formulation in transdermal patch makes it particularly suitable for use in the elderly with chronic non-malignant pain. In a study of 93 frail patients with a mean age of 79.7 years and a mix of nociceptive and mixed chronic pain, low to medium doses of transdermal buprenorphine during a three month period significantly reduced pain intensity and improved sleep quality versus baseline without adverse central nervous system (CNS) side effects orcognitive changes as measured by the Mini-Mental State Examination (MMSE). Furthermore side effects caused treatment withdrawal in only $13 \%$ of patients ${ }^{[53]}$. Another study included 891 patients with chronic non-malignant pain (with a neuropathic component present in $69 \%$ of cases) and a mean age of 72.8 years $(69 \%$ of them were over 70$)$. Treatment with LDTB for 12 weeks had a positive effect in theis mostly elderly patientpopulation; most experienced improvements their capacity to perform daily activities and quality of life and sustained pain relief, leading to a substantial reduction in the overall burden of pain ${ }^{[20]}$.

Unlike other opioids, the phamacological characteristics of transdermal buprenorphine offer safe and effective pain relief to the elderly with no need for dose adjustment ${ }^{[19,54]}$. During normal aging many different metabolic changes develop that can have a clear impact in the pharmacokinetic profile of a drug. These changes were evaluated for buprenorphine in 72 healthy individuals. No differences in the pharmacokinetics of LDTB were observed when a 50 to 60 year-old group was compared to a $>75$ year-old group ${ }^{[55]}$.

One essential advantage of LDTB in the elderly population is the transdermal route. The incidence of dysphagia increases with age, and dysphagia rates in older adults can be as high as $60 \% \%^{[56]}$. this is a problem in institucionalized people as between $15 \%$ and $33 \%$ of patients in nursing homes report having swallowing difficulties in relation to taking solid oral medications $^{[56]}$

\section{Summary}

Low-dose transdermal buprenorphine can be a good option for the management of chronic non-cancer pain, Table 1 highlights the various reasons that support this statement. 
TABLE 1: Buprenorphine attributes.

Proven and reliable safety and efficacy for moderate/severe pain in various types of non-cancer painful conditions

Long duration of action resulting in sustained pain relief

Multiple dose options allows for tailoring to patients needs

Dosing flexibility and 7 day application

Potential antihyperalgesic effects

Respiratory-depression ceiling effect

Fewer typical opioid side effects

Titration may improve tolerability

Lower abuse and addiction potential

Can be used safely in patients with renal dysfunction and poor hepatic function - good safety profile with elderly

Nausea and Vomiting side effects can potentially be avoided or managed

Simple and convenient; Improvements in ADL, QoL, and Function

*ADL- Activities of Daily Living and QoL- Quality of Life.

\section{Conclusion}

Chronic non-cancer pain is prevalent in Mexico, with an increasing incidence due to the aging population, In light of the wealth of treatment options, clinicians must carefully weigh risks against benefits when selecting individual pharmacotherapy for pain control. Opioids are safe, effective, and important analgesic agents in the pharmacologic treatment of chronic pain, in particular as NSAID safety appears to be questionable for long-term use and may be contraindicated in patients with heart disease, renal dysfunction, or those with risk factors for gastrointestinal disorders.Likewise, opioidshave their own risks and benefits. In this context, it is worth considering buprenorphine - an opioid agent with certain unique pharmacological characteristics - particularly in a low-dose transdermal formulation.

Favorable characteristics of buprenorphine include morphine-equivalent clinical efficacy, a ceiling effect for respiratory depression, lower abuse liability relative to other opioids, and good tolerability. In the transdermal formulation, buprenorphine offers effective round-the-clock analgesia in a clinically convenient formulation that reduces issues of patient compliance. So for those healthcare providers who are familiar with buprenorphine, this is an additional option to provide good analgesia for moderate chronic non-cancer pain. For those who are not currently prescribing buprenorphine, the low-dose transdermal formulation introduces a new option for their patients who have moderate chronic non-cancer pain, one that can be considered for use in place of long term NSAIDs, COXIBs, tramadol, or tramadol combinations.

\section{References}

1. WHO's Cancer pain ladder for adults. World Health Organization. 2. AGS Clinical Practice Guideline: Pharmacological management of persistent pain in older persons. (2013) American Geriatrics Society.

3. Auret, K., Schug, S.A. Underutilisation of opioids in elderly patients with chronic pain: approaches to correcting the problem. (2005) Drugs aging 22(8): 641-654.

4. Rhodin, A. The rise of opiophobia: is history a barrier to prescribing? (2006) J pain palliat care pharmacother 20(3): 31-32.
5. Chang, G., Chen, L., Mao, J. Opioid tolerance and hyperalgesia. (2007) Med Clin North Am 91(2): 199-211.

6. Lee, M., Silverman, S.M., Hansen, H., et al. A comprehensive review of opioid-induced hyperalgesia. (2011) Pain physician 14(2): 145-161. 7. DIC. Close Up Audit. 2014.

8. Roberts, E., Delgado Nunes, V., Buckner, S., et al. Paracetamol: not as safe as we thought? A systematic literature review of observational studies. (2016) Ann Rheum Dis 75(3): 552-559.

9. Bhala, N., Emberson, J., Merhi, A., et al. Vascular and upper gastrointestinal effects of non-steroidal anti-inflammatory drugs: meta-analyses of individual participant data from randomised trials. (2013) Lancet 382(9894): 769-779

10. Pergolizzi, J., Aloisi, A.M., Dahan, A., et al. Current knowledge of buprenorphine and its unique pharmacological profile. (2010) Pain Pract 10(5): 428-450.

11. Koppert, W., Ihmsen, H., Korber, N., et al. Different profiles of buprenorphine-induced analgesia and antihyperalgesia in a human pain model. (2005) Pain 118(1-2): 15-22.

12. Hovell, B.C., Ward, A.E. Pain relief in the post-operative period: a comparative trial of morphine and a new analgesic buprenorphine. (1977) J Int Med Res 5(6): 417-421.

13. Christoph, T., Kogel, B., Schiene, K., et al. Broad analgesic profile of buprenorphine in rodent models of acute and chronic pain. (2005) Eur J Pharmacol 507(1-3): 87-98.

14. Raffa, B.R., Ding, Z. Examination of the preclinical antinociceptive efficacy of buprenorphine and its designation as full- or partial-agonist. (2007) Acute Pain 9(3): 145-152.

15. Greenwald, M.K., Johanson, C.E., Moody, D.E., et al. Effects of buprenorphine maintenance dose on mu-opioid receptor availability, plasma concentrations, and antagonist blockade in heroin-dependent volunteers. (2003) Neuropsychopharmacology 28(11): 2000-2009.

16. Ding, Z., Raffa, R.B. Identification of an additional supraspinal component to the analgesic mechanism of action of buprenorphine. (2009) Br J Pharmacol 157(5): 831-843.

17. Walsh, S.L., Preston, K.L., Stitzer, M.L., et al. Clinical pharmacology of buprenorphine: ceiling effects at high doses. (1994) Clin Pharmacol Ther 55(5): 569-580.

18. Pergolizzi, J., Boger, R.H., Budd, K., et al. Opioids and the management of chronic severe pain in the elderly: consensus statement of an International Expert Panel with focus on the six clinically most often used World Health Organization Step III opioids (buprenorphine, fentanyl, hydromorphone, methadone, morphine, oxycodone). (2008) Pain pract 8(4): 287-313.

19. Likar, R., Vadlau, E.M., Breschan, C., et al. Comparable analgesic efficacy of transdermal buprenorphine in patients over and under 65 years of age. (2008) Clin J Pain 24(6): 536-543.

20. Uberall, M.A., Muller-Schwefe, G.H. Low-dose 7-day transdermal buprenorphine in daily clinical practice - perceptions of elderly patients with moderate non-malignant chronic pain. (2012) Curr Med Res Opin 28(10): 1585-1595.

21. Kress, H.G. Clinical update on the pharmacology, efficacy and safety of transdermal buprenorphine. (2009) Eur J Pain 13(3): 219-230.

22. Sittl, R., Nuijten, M., Nautrup, B.P. Changes in the prescribed daily doses of transdermal fentanyl and transdermal buprenorphine during treatment of patients with cancer and noncancer pain in Germany: results of a retrospective cohort study. (2005) Clin Ther 27(7): 1022-1031. 23. Mercadante, S., Ferrera, P., Villari, P., et al. Frequency, indications, outcomes, and predictive factors of opioid switching in an acute palliative care unit. (2009) J pain symptom manage 37(4): 632-641.

24. Hans, G., Robert, D. Transdermal buprenorphine - a critical appraisal of its role in pain management. (2009) J Pain Res 2: 117-134.

25. Plosker, G.L. Buprenorphine 5, 10 and $20 \mathrm{ug} / \mathrm{h}$ transdermal patch: a review of its use in the management of chronic non-malignant pain. (2011) Drugs 71(18): 2491-2509.

26. Landau, C.J., Carr, W.D., Razzetti, A.J., et al. Buprenorphine transdermal delivery system in adults with persistent noncancer-related pain syndromes who require opioid therapy: a multicenter, 5-week run-in 
and randomized, double-blind maintenance-of-analgesia study. (2007) Clin Ther 29(10): 2179-2193.

27. Przeklasa-Muszynska, A., Dobrogowski, J. Transdermal buprenorphine for the treatment of moderate to severe chronic pain: results from a large multicenter, non-interventional post-marketing study in Poland. (2011) Curr Med Res Opin 27(6): 1109-1117.

28. Griessinger, N., Sittl, R., Likar, R. Transdermal buprenorphine in clinical practice--a post-marketing surveillance study in 13,179 patients. (2005) Curr Med Res Opin 21(8): 1147-1156.

29. Leng, X., Li, Z., Lv, H., et al. Effectiveness and Safety of Transdermal Buprenorphine Versus Sustained-Release Tramadol in Patients with Moderate to Severe Musculoskeletal Pain: An 8-Week, Randomized, Double Blind, Double Dummy, Multi-Center, Active-controlled, Non-inferiority Study. (2015) Clin J pain 31(7): 612-620.

30. Breivik, H., Collett, B., Ventafridda, V., et al. Survey of chronic pain in Europe: prevalence, impact on daily life, and treatment. (2006) Eur J pain 10(4): 287-333.

31. Gallagher, A.M., Leighton-Scott, J., van Staa, T.P. Utilization characteristics and treatment persistence in patients prescribed low-dose buprenorphine patches in primary care in the United Kingdom: a retrospective cohort study. (2009) Clin Ther 31(8):1707-1715.

32. Munera, C., Drehobl, M., Sessler, N.E., et al. A randomized, placebo-controlled, double-blinded, parallel-group, 5-week study of buprenorphine transdermal system in adults with osteoarthritis. (2010) J Opioid manag 6(3): 193-202.

33. Karlsson, M., Berggren, A.C. Efficacy and safety of low-dose transdermal buprenorphine patches $(5,10$, and $20 \mathrm{microg} / \mathrm{h})$ versus prolonged-release tramadol tablets $(75,100,150$, and $200 \mathrm{mg})$ in patients with chronic osteoarthritis pain: a 12-week, randomized, open-label, controlled, parallel-group noninferiority study. (2009) Clin ther 31(3): 503-513.

34. Conaghan, P.G., O’Brien, C.M., Wilson, M., et al. Transdermal buprenorphine plus oral paracetamol vs an oral codeine-paracetamol combination for osteoarthritis of hip and/or knee: a randomised trial. (2011) Osteoarthritis cartilage 19(8): 930-938.

35. Frymoyer, J.W., Cats-Baril, W.L. An overview of the incidences and costs of low back pain. (1991) Orthop Clin North Am 22(2): 263-271.

36. Gordon, A., Callaghan, D., Spink, D., et al. Buprenorphine transdermal system in adults with chronic low back pain: a randomized, double-blind, placebo-controlled crossover study, followed by an open-label extension phase. (2010) Clin Ther 32(5): 844-860.

37. Miller, K., Yarlas, A., Wen, W., et al. The impact of buprenorphine transdermal delivery system on activities of daily living among patients with chronic low back pain: an application of the international classification of functioning, disability and health. (2014) Clin J Pain 30(12): 1015-1022.

38. Yarlas, A., Miller, K., Wen, W., et al. A randomized, placebo-controlled study of the impact of the 7-day buprenorphine transdermal system on health-related quality of life in opioid-naive patients with moderate-to-severe chronic low back pain. (2013) J Pain 14(1): 14-23. 39. Yarlas, A., Miller, K., Wen, W., et al. Buprenorphine Transdermal System Improves Sleep Quality and Reduces Sleep Disturbance in $\mathrm{Pa}$ tients with Moderate-to-Severe Chronic Low Back Pain: Results from Two Randomized Controlled Trials. (2015) Pain Pract.

40. Steiner, D., Munera, C., Hale, M., et al. Efficacy and safety of buprenorphine transdermal system (BTDS) for chronic moderate to severe low back pain: a randomized, double-blind study. (2011) J Pain 12(11): 1163-1173.
41. Huffman, K.L., Shella, E., Sweis, G., et al. Non-opioid substance use disorders and opioid dose predict therapeutic opioid addiction. (2015) J Pain 16(2): 126-134.

42. Raffa, R.B., Pergolizzi, J.V.Jr. Is morphine medicine's biggest misnomer? (2012) Ann pharmacy other 46(7-8): 1122-1123.

43. Anghelescu, D.L., Ehrentraut, J.H., Faughnan, L.G. Opioid misuse and abuse: risk assessment and management in patients with cancer pain. (2013) J Natl Compr Canc Netw 11(8): 1023-1031.

44. Wunsch, M.J., Cropsey, K.L., Campbell, E.D., et al. OxyContin use and misuse in three populations: substance abuse patients, pain patients, and criminal justice participants. (2008) J Opioid Manage 4(2): 73-79. 45. Brennan, M.J., Stanos, S. Strategies to optimize pain management with opioids while minimizing risk of abuse. (2010) PM R 2(6): 544558.

46. Atluri, S., Akbik, H., Sudarshan, G. Prevention of opioid abuse in chronic non-cancer pain: an algorithmic, evidence based approach. (2012) Pain physician 15(3 Suppl): ES177-189.

47. Comer, S.D., Sullivan, M.A., Whittington, R.A., et al. Abuse liability of prescription opioids compared to heroin in morphine-maintained heroin abusers. (2008) Neuropsychopharmacology 33(5): 1179-1191.

48. Ducharme, S., Fraser, R., Gill, K. Update on the clinical use of buprenorphine: in opioid-related disorders. (2012) Can Fam physician 58(1): $37-41$

49. Office of Diversion Control. Schedules of Controlled Substances: Rescheduling of Buprenorphine from Schedule V to Schedule III. (2002) Drug Enforcement Administration 67(194).

50. Moore, R.A., McQuay, H.J. Prevalence of opioid adverse events in chronic non-malignant pain: systematic review of randomised trials of oral opioids. (2005) Arthritis Res Ther 7(5): R1046-R1051.

51. James, I.G., O’Brien, C.M., McDonald, C.J. A randomized, double-blind, double-dummy comparison of the efficacy and tolerability of low-dose transdermal buprenorphine (BuTrans seven-day patches) with buprenorphine sublingual tablets (Temgesic) in patients with osteoarthritis pain. (2010) J Pain Symptom Manage 40(2): 266-278.

52. Wen, W., Lynch, S.Y., Munera, C., et al. Application site adverse events associated with the buprenorphine transdermal system: a pooled analysis. (2013) Expert Opin Drug Saf 12(3): 309-319.

53. Gianni, W., Madaio, A.R., Ceci, M., et al. Transdermal buprenorphine for the treatment of chronic noncancer pain in the oldest old. (2011) J Pain Symptom Manage 41(4): 707-714.

54. Filitz, J., Griessinger, N., Sittl, R., et al. Effects of intermittent hemodialysis on buprenorphine and norbuprenorphine plasma concentrations in chronic pain patients treated with transdermal buprenorphine. (2006) European Journal of Pain (London, England) 10(8): 743-748.

55. Al-Tawil, N., Odar-Cederlof, I., Berggren, A.C., et al. Pharmacokinetics of transdermal buprenorphine patch in the elderly. (2013) Eur J Clin Pharmacol 69(2): 143-149.

56. Morris, H. Dysphagia in the elderly--a management challenge for nurses. (2006) Br J Nurs 15(10): 558-562.
Ommega Online Publishers

Journal Title: Journal of Anesthesia and Surgery (JAS)

Journal Short Name: J Anesth Surg
Journal ISSN: 2377-1364

E-mail: anestheisa@ommegaonline.com

Website: www.ommegaonline.org 